\section{Radikulärer Kreuzschmerz: Gabapentin und Steroide gleichauf}

\begin{abstract}
Das Antikonvulsivum Gabapentin wird auch zur Therapie neuropathischer Schmerzen eingesetzt. Wie viel sich mit Gabapentin gegen diese Art von Kreuzweh ausrichten lässt, verglichen mit epiduralen Steroidinjektionen, haben US-amerikanische Schmerzmediziner untersucht.
\end{abstract}

ine neuropathische Komponente do-
folge bei $17-55 \%$ der Patienten mit chro-
nischen radikulären Schmerzen im un-
teren Bereich des Rückens. 145 Patienten
mit lumbosakraler Radikulopathie, de-
ren Beinschmerzen mindestens so stark
waren wie jene im Rücken, waren an der
Studie beteiligt. Ursache der Symptome
waren ein Diskusprolaps oder eine Ste-
nose des Spinalkanals, die Beschwerden
bestanden seit weniger als 4 Jahren. 73
Patienten bekamen eine epidurale Stero-
idinjektion ( 60 mg Methylprednisolon, 1
ml Bupivacain $0,25 \%)$ plus Placebo ana-
log zum Schema von Gabapentin, 72 er-
hielten eine Scheininjektion und Gaba-
pentin oral (Kapseln zu 300 mg, Zieldo-
sis $1.800-3.600 \mathrm{mg} /$ Tag, auftitriert über

15-24 Tage, mindestens 5 Tage Behandlung mit therapeutischer Dosis vor dem Follow up).

1 Monat nach Therapiebeginn war im primären Endpunkt kein Unterschied zwischen den beiden Gruppen festzustellen. Auf einer Schmerzskala (numerische Rating-Skala) von 0 bis 10 (stärkste Schmerzen) lag der Wert von Patienten nach Steroidinjektion im Mittel bei 3,3, der Rückgang gegenüber dem Ausgangswert betrug durchschnittlich 2,2 Punkte. Mit Gabapentin erreichte der Schmerzwert 3,7 Punkte, im Mittel 1,7 Punkte weniger als zu Beginn der Studie.

Ganz ähnlich sah es auch nach 3 Monaten aus. Die Scores der Patienten nach epiduraler Steroidinjektion $(3,4 /-2,0)$ und nach Gabapentin $(3,7 /-1,6)$ lagen dicht beieinander. Ein statistisch relevanter Unterschied war nicht zu erkennen.

Die Ergebnisse in einigen sekundären Endpunkten fielen eher zugunsten der Steroidinjektion aus. So war in dieser Gruppe der Rückgang in der Rubrik „schlimmste Beinschmerzen“ mit 3,0 Punkten deutlicher ausgeprägt als bei Gabapentin $(-2,0)$, aber nur nach dem ersten Monat. Auch ein positives Ergebnis - definitionsgemäß ein Rückgang des Schmerzwertes um mindestens 2 Punkte - wurde nach 1 Monat häufiger mit der epiduralen Injektion erreicht (66 vs. $46 \%$ unter Gabapentin).

Fazit: Es möge schon sein, dass epidurale Steroidinjektionen in mancher Hinsicht einen größeren Nutzen bei radikulären Lumbosakralschmerzen einbrächten, schreiben die Forscher. „Aber die Unterschiede sind gering und zudem für die meisten Patienten vorübergehender Natur."

Robert Bublak

Cohen SP et al. Epidural steroid injections compared with gabapentin for lumbosacral radicular pain: multicenter randomized double blind comparative efficacy study. BMJ. 2015;350:h1748.

\title{
Demenzkranke leiden oft unerkannt unter Schmerzen
}

\section{Schmerzen bei Demenzkranken treten häufig auf. Trotzdem bleiben sie meist un(ter)behandelt, da sie sich statt mit Rastlosigkeit eher durch pathologisches Verhalten äußern.}

S chmerzen bei Demenz verhindern die Heilung und Genesung, wenn sie unbehandelt bleiben, lösen funktionale Einschränkungen aus und fördern das Auftreten von Verhaltensstörungen und psychiatrischen Symptomen bei Demenz (BPSD).

Britische Forscher untersuchten deshalb in einer longitudinalen Kohortenstudie 230 über 70 -jährige Patienten, die wegen ihrer Demenz und nicht geplanten ärztlichen Eingriffen an 2 verschiedenen Allgemeinkrankenhäusern eingewiesen wurden. Die Teilnehmer der Studie wurden bei der Aufnahme und alle 4 weiteren Tage nach ihren Schmerzen befragt (mit einfachen Ja- und Nein- Fragen und den Gesichtsskalen für Schmerzen) sowie mit der Fremdbeobachtungsskala für Schmerzen bei fortgeschrittener Demenz („Pain Assessment in Advanced Dementia scale“, PAINAD) bei Ruhe und Bewegung. Des Weiteren wurde der CohenMansfield-Beobachtungsbogen für Agitation und der Test für die Untersuchung von BPSD eingesetzt („Behavioural Pathology in Alzheimer Disease Scale“, BEHAVE-AD). Bei der Aufnahme litt jeder vierte Demenzkranke (27\%) an Schmerzen und im Laufe der Untersuchung stieg dieser Anteil sogar bei mindestens einer Gelegenheit auf 39\%. Die Hälfte der Patienten konnte die Gesichtsskala ausfüllen, bei fortgeschrittener Demenz gelang dies nicht mehr. Nach PAINAD hatte jeder fünfte (19\%) Ruheschmerzen und über die Hälfte (57\%) hatte bei Bewegung bei mindestens einer Gelegenheit
Schmerzen. Bei jedem sechsten (16\%) hielt der Schmerz bei der gesamten Untersuchung an. Die detaillierte Analyse zeigte, dass Schmerzen bei Ruhe und Bewegung nicht mit Agitation korrelierten, aber stark assoziiert waren mit einem hohen BEHAVE-AD-Score. Am deutlichsten ausgeprägt war die Verbindung zu Aggression und Ängstlichkeit.

Fazit: Schmerzen sind bei Patienten mit Demenz, die stationär behandelt werden müssen, sehr häufig und äußern sich mit BPSD. Ein verbessertes Schmerzmanagement könnte Ängste und Aggressionen reduzieren und die Qualität der stationären Krankenversorgung steigern.

Stephanie Kraus

Sampson EL et al. Pain, agitation, and behavioural problems in people with dementia admitted to general hospital wards: a longitudinal cohort study. Pain. 2015;156(4):675-83. 